\title{
SITUACIÓN ECONÓMICA, POLÍTICA FISCAL Y PANDEMIA EN COLOMBIA*
}

Jorge Armando Rodriguez

* DOI: https://doi.org/10.18601/01245996.v23n44.12. Este escrito integra, actualiza y amplía varias notas periodísticas del autor, en especial el artículo publicado en UN Periódico, 226, en julio pasado. Recepción: 17-09-2020, modificación final: 12-11-2020, aceptación: 27-11-2020. Sugerencia de citación: Rodríguez, J. A. (2021). Situación económica, política fiscal y pandemia en Colombia. Revista de Economia Institucional, 23(44), 249-263.

a Decano, Facultad de Ciencias Económicas, Universidad Nacional de Colombia. Bogotá, Colombia, [jarodrigueza@unal.edu.co], [https://orcid. org/0000-0002-6940-8990]. 


\section{Situación económica, política fiscal y pandemia en Colombia}

Resumen Este artículo analiza la evolución de la economía colombiana durante la pandemia de COVID-19 desde una perspectiva agregada, así como la política fiscal adoptada para enfrentar la crisis desatada por la enfermedad. Argumenta que en la coyuntura actual el objetivo principal de la política macroeconómica debería ser aumentar el nivel de empleo y que la austeridad fiscal adoptada por el gobierno nacional no lleva a ese fin. Además, considera el papel de la tributación en este contexto y examina los programas que el gobierno emprendió en uso de facultades de emergencia a la luz de la necesidad de un marco económico e institucional que ayude a enfrentar eficazmente crisis socioeconómicas y desastres naturales.

Palabras clave: economía de la COVID, economía colombiana, política fiscal; JEL: I18, E02, E69

\section{Economic situation, fiscal policy and pandemic in Colombia}

Abstract This article analyzes the evolution of the Colombian economy during the COVID-19 pandemic from an aggregate perspective, as well as the fiscal policy adopted to face the crisis unleashed by the disease. It argues that in the current situation the main objective of macroeconomic policy should be to increase the level of employment and that the fiscal austerity adopted by the national government does not lead to that end. Furthermore, it considers the role of taxation in this context, and examines the government programs undertaken in use of emergency powers in light of the need for an economic and institutional framework that helps to effectively deal with socio-economic crises and natural disasters.

Keywords: COVID economics, Colombian economy, fiscal policy;JEL: I18, E02, E69

\section{Situação econômica, política fiscal e pandemia na Colômbia}

Resumo Este artigo analisa a evolução da economia colombiana durante a pandemia COVID-19 de uma perspectiva agregada, bem como a política fiscal adotada para enfrentar a crise desencadeada pela doença. Ele argumenta que na situação atual o principal objetivo da política macroeconômica deveria ser o aumento do nível de emprego e que a austeridade fiscal adotada pelo governo nacional não leva a esse fim. Além disso, considera o papel da tributação neste contexto e examina os programas que o governo empreendeu no uso de poderes de emergência à luz da necessidade de um quadro econômico e institucional que ajude a enfrentar com eficácia as crises socioeconômicas e desastres naturais.

Palavras-chave: economia COVID, economia colombiana, política fiscal; JEL: I18, E02, E69 
P ara contener la propagación de la pandemia de COVID-19, en Bogotá se hizo un simulacro de aislamiento pedagógico y a finales de marzo se estableció la cuarentena en todo el país. La pandemia y las medidas de distanciamiento físico para contenerla afectaron la economía colombiana, pues redujeron las actividades de producción y distribución, cercenaron la demanda agregada y acentuaron la fragilidad financiera. La crisis económica ocasionada por esta combinación inusual de circunstancias ha perjudicado tanto a los trabajadores como a los propietarios de capital, probablemente agravando la desigual distribución del ingreso y de la riqueza.

Las actividades económicas se empezaron a recuperar con el fin de la cuarentena y el inicio del aislamiento selectivo en septiembre de 2020, pero el tipo de recuperación es incierto. Se han planteado varios escenarios, dependiendo de que las pérdidas de producto, empleo e ingreso sean temporales o permanentes, de la forma que asuma la reactivación (en forma de $\mathrm{V}, \mathrm{U}, \mathrm{W}$ o L) y de la intensidad de los posibles rebrotes de la enfermedad. Con respecto a los escenarios más optimistas cabe recordar una advertencia de Joan Robinson: "Puede que se necesiten dos meses para obtener respuesta a una carta dirigida a una dependencia gubernamental, pero una industria privada tarda veinte años en reajustarse a una caída de la demanda" (1978, p. 27). Aunque las economías de China y de Estados Unidos parecen recuperarse con rapidez, América Latina es un epicentro de la pandemia y sufre la peor recesión de su historia, como señala Ocampo (2020); la caída estimada de la actividad económica en la región será de un 9\% en 2020, con efectos muy adversos en el país. La recesión económica va más allá de la menor utilización de la capacidad instalada. Si bien en toda recesión se cierran empresas, la destrucción del tejido empresarial ha sido extraordinaria, como también lo ha sido la pérdida de empleos e ingresos de los trabajadores. Al margen de cuánto se tarde para volver a los niveles de actividad anteriores, el camino hacia la recuperación luce sinuoso y nada expedito (CID, 2020).

La pandemia restringe la eficacia potencial de las políticas macroeconómicas que buscan superar la recesión, así sean adecuadas. Las medidas de distanciamiento físico, entre ellas los límites a las aglomeraciones, adoptadas para atenuar los contagios, afectan el funcionamiento del mercado. El miedo a la enfermedad, intensificado por la inexistencia de una vacuna, actúa en el mismo sentido. "Según los historiadores, las pandemias suelen tener dos tipos de finales: el médico, que se produce cuando las tasas de incidencia y de mortalidad 
se desploman, y el social, cuando la epidemia de miedo a la enfermedad disminuye" (Kolata, 2020).

Ante esta situación, el objetivo central de las políticas macroeconómicas -fiscal y monetaria- debería ser elevar el nivel de empleo, la fuente principal de ingresos de los hogares. $\mathrm{El}$ aumento del desempleo, en especial entre jóvenes y mujeres, ha sido dramático y más agudo en unos departamentos y ciudades que en otros. Además, la Constitución establece que el Estado "de manera especial, intervendrá para dar pleno empleo a los recursos humanos" (CPC, art. 334). Keynes argumentó en forma convincente que una de las principales fallas "de la sociedad económica en la que vivimos [es] su incapacidad para procurar la ocupación plena” (1965, p. 328). Siguiendo una práctica común de décadas recientes, el crecimiento económico es uno de los objetivos centrales del gobierno Duque, objetivo que -al margen de lo que se piense sobre su importancia- no se menciona en la carta política, a diferencia del nivel de empleo (Rodríguez, 2018). El Plan Nacional de Desarrollo 2018-2022 buscaba acelerar el crecimiento económico, del 2,7\% en 2018 al 4,5\% en 2022 (DNP, 2019, pp. 1055-1056). Acaecida la pandemia, ese objetivo se mantiene: "E1 escenario central del Gobierno se fundamenta en la recuperación del crecimiento económico", dice el Marco Fiscal de Mediano Plazo (Minhacienda, 2020a, p. 273), aunque las metas y proyecciones específicas hayan cambiado. Las estimaciones oficiales ahora indican un crecimiento negativo del $5,5 \%$ en 2020 , y sugieren un crecimiento del 6,6\% en 2021 (ibíd., p. 26).

De manera inusual, el mismo gobierno también se fijó como objetivo reducir el desempleo. Según el Plan Nacional de Desarrollo, la meta era llegar a un desempleo del 7,9\% en 2022 (DNP, 2019, p. 1065). En medio de la pandemia, el gobierno nacional ratificó esa meta (Minhacienda, 2020b, pp. 25-26). El nivel de empleo y el crecimiento económico pueden estar correlacionados, acaso como describe Okun (1962), pero no son idénticos y no siempre van en la misma dirección.

¿Hay congruencia entre ese objetivo del nivel de empleo y los medios propuestos para lograrlo? Una respuesta breve sería no o, en el mejor de los casos, poca. Combatir el alto desempleo actual requiere una política fiscal expansiva, pero el gobierno del presidente Duque prefiere adoptar una política de austeridad. Así, según el Marco Fiscal de Mediano Plazo, "en el año 2021 se llevará a cabo un ajuste sustancial del déficit fiscal, desmontando buena parte de los gastos extraordinarios de la emergencia" (Minhacienda, 2020a, p. 23). Y anuncia que desde 2022 la austeridad será más severa. Esa política 
es contraproducente y va en contravía de la creación de empleos y de la mejora de los ingresos de los hogares.

La inflación anual es inferior a la meta de mediano plazo del 3\% establecida por el Banco de la República, y en septiembre de 2020 se situó por debajo del límite inferior del rango meta (el 2\%). Puesto que la estabilidad de precios es un objetivo constitucional prioritario del banco central, pero no exclusivo, estas cifras indican que es posible y oportuno adoptar - para lo que resta de este año, en 2021 y quizás más adelante- una política monetaria expansiva cuyo objetivo sea contribuir a la reducción del alto desempleo.

En una dirección acorde con la necesidad de reactivar la economía, el Banco de la República ha reducido de modo gradual la tasa de interés de intervención, del 4,25\% a finales de marzo al 1,75\% a finales de septiembre. También ha proporcionado liquidez reduciendo el encaje bancario y comprado títulos de deuda pública y privada. Pero todo ello puede ser insuficiente. Aparte del margen que pueda haber para reducir aún más la tasa de intervención, con el fin de ampliar la demanda agregada y contribuir a lograr el objetivo de empleo no se debería descartar la opción de que el banco otorgue financiamiento directo al gobierno nacional. En general, no se deben descartar por principio - sin tener en cuenta las circunstancias- las políticas monetarias no convencionales.

Además del desempleo y de la pobreza monetaria asociada, es probable que la pandemia haya empeorado la distribución del ingreso. Con base en datos históricos de las epidemias y pandemias ocurridas desde la gran peste de 1348, Sayed y Peng (2020) sostienen que su impacto directo en la distribución del ingreso es el resultado de que los grupos de personas pobres y de bajos ingresos son los más vulnerables a la propagación de los virus mortales. En Colombia, hasta el momento el 67,6\% de las víctimas de la COVID-19 son personas de estratos socioeconómicos 1 y 2 (Dane,2020a), estratos que constituyen el $72 \%$ de la población. Además, las tasas de mortalidad más altas se registran entre los hombres y las personas mayores de 60 años.

Sin embargo, Sayed y Peng encuentran que algunas pandemias llevaron a una reducción de la desigualdad de ingresos, y señalan que el efecto final sobre la desigualdad dependió ante todo de las características de la enfermedad. Pero, no hay acuerdo sobre esta conclusión y el tema no está zanjado. Por ejemplo, según Alfani (2020) las pandemias posteriores a la peste negra del siglo XIV no provocaron una reducción de la desigualdad en el reparto de la riqueza "debido a entornos institucionales y efectos del mercado laboral diferentes” $y$, en 
su opinión, algo similar ocurrirá con la pandemia actual. Teniendo en cuenta que las muertes por COVID-19 se concentran en los grupos de más edad, "no podemos esperar una escasez de mano de obra ni una fuerte caída de la productividad", sostienen Sayed y Peng (2020, p. 96). Estos autores predicen altas tasas de desempleo, una reducción del consumo y un aumento de las deudas. "Los efectos finales sobre la desigualdad no son claros todavía”, si bien algunas de las características de la enfermedad la empeoran (ibíd.).

Pero no todo se reduce a las características de la enfermedad.

Los tiempos difíciles y los periodos de agitación social serían, en forma paradójica, propicios para establecer o consolidar un marco institucional y económico que responda eficazmente a las crisis socioeconómicas y los desastres naturales. $\mathrm{O}$, al menos, para aumentar el gasto público necesario para enfrentarlos, como sostienen Peacock y Wiseman (1961). En nuestro país ese marco hoy se encuentra en un estado embrionario y, por ello, los costos de la pandemia son muy altos, y pueden aumentar cuando se extiendan y profundicen las consecuencias de la pandemia. Sin considerar los posibles efectos de las respuestas de política, un ejercicio del Observatorio de coyuntura económica y social (Cede, 2020) concluye que, como resultado de la pandemia, "se podría producir un efecto en pobreza y desigualdad equivalente a retroceder a los inicios del siglo XXI".

Sea como fuere, y pese a ciertos avances, la protección social que brinda el Estado social de derecho al que aspira la Constitución es aún lejana. Después de la reciente revisión del Dane (2020b), hoy se sabe que la pobreza monetaria es mayor de lo que se creía (p. ej., el porcentaje de población pobre en 2019 era el 35,7\%, y no el 27\%). Cerca de la mitad de la población en edad de jubilación no está cubierta por el sistema de pensiones, y entre los afiliados a los fondos privados más del $80 \%$ no reunirá el capital suficiente para jubilarse ${ }^{1}$.

La pandemia ha puesto a prueba la capacidad de las instituciones para proteger a los miembros de la sociedad que están en peores condiciones para hacerle frente. Desde el comienzo fue evidente la falta de mecanismos para canalizar recursos hacia vendedores ambulantes, empleadas domésticas, jornaleros y destechados, por ejemplo. La respuesta del gobierno fue crear, en medio de la crisis, programas como Ingreso Solidario, que han tenido grandes dificultades para identificar a los beneficiarios potenciales. También sometió a prueba la capacidad del sector público para promover la creación de empleos, bien sea en

1 Farné, Rodríguez y Ríos (2017) muestran el panorama del sistema de pensiones.

Revista de Economía Institucional, vol. 23 , N. ${ }^{\circ} 44$, Primer semestre/202i, pp. $249-263$ ISSN OI 24-5996/E-ISSN 2346-2450 
forma indirecta, a través de la empresa privada (estímulos a la demanda agregada, incentivos a la inversión y subsidios), o directa, como empleador de última instancia, una opción que ha propuesto Wray (2007) para los países en desarrollo y Gómez (2020) para nuestro país.

\section{MAGNITUD DE LOS PROBLEMAS}

En comparación con la situación anterior al estado de emergencia, declarado a mediados de marzo de 2020, el desempleo se ha disparado. Debido a la pandemia, a la cuarentena y otras medidas para contenerla, la compra y venta de mercancías se ha visto obstaculizada, no solo en términos físicos, golpeando los ingresos del trabajo y del capital, excepto quizá en las actividades que buscan proteger la salud y asegurar la supervivencia, como las de los médicos, hospitales y farmacéuticas, y la provisión de alimentos.

Según el Dane, la situación laboral más grave se registró en mayo, cuando la población ocupada se redujo a 17,3 millones, un $22 \%$ menos que un año antes. La pérdida de empleos fue severa entre marzo y mayo, cuando el desempleo pasó del 12,6 al 21,4 \%. Mucha gente desistió de buscar empleo durante el aislamiento preventivo y engrosó la población económicamente inactiva; así, la tasa de desempleo solo capta en forma parcial el empeoramiento de las condiciones laborales. $\mathrm{Si}$ los nuevos inactivos hubieran seguido buscando trabajo, esa tasa habría llegado a un 30\%. Aunque empezó a descender (en septiembre fue del 15,8\%), se ha mantenido, mes a mes, muy por encima del nivel del año anterior (gráfica 1).

Gráfica 1

Tasa de desempleo mensual

(Total nacional)

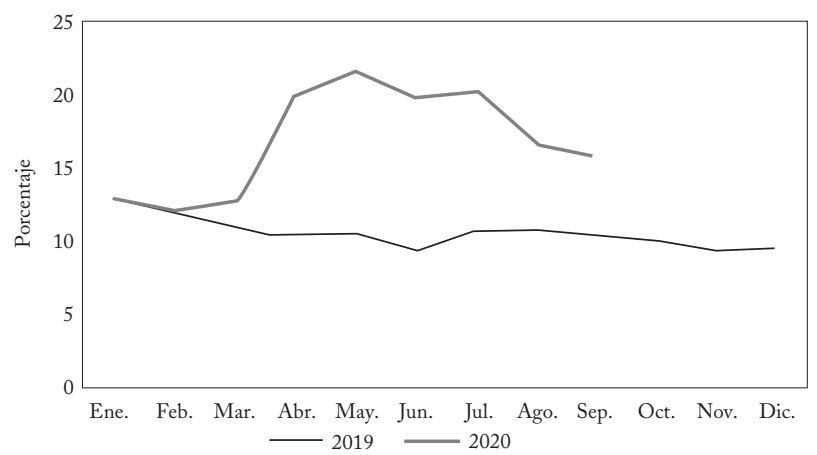

Fuente: Dane [www.dane.gov.co/index.php/estadisticas-por-tema/mercado-laboral/empleo-y-desempleo]. 
E1 desempleo suele variar de manera ostensible en términos territoriales, y así ha ocurrido durante la pandemia. La información hoy disponible se refiere a las ciudades y áreas metropolitanas. En todas la desocupación primero aumentó y luego disminuyó; la diferencia de la tasa de desempleo entre la ciudad más golpeada y la menos afectada supera los 15 puntos porcentuales. En el trimestre julio-septiembre fue del 27,5\% en Ibagué y del 12,3\% en Barranquilla (en el mismo trimestre de 2019, en Ibagué fue del 13,6\%, solo 5,7 puntos porcentuales superior a la de Barranquilla). Algo similar sucede con el desempleo por sexo y edad. En julio-septiembre de 2020, la tasa de desempleo de las mujeres llegó al 22,8\%, y la de los hombres fue del 13,9\%. Con respecto a ese mismo trimestre de 2019, el desempleo aumentó algo más de 9 puntos porcentuales entre las mujeres y cerca de 6 puntos entre los hombres. Y durante el trimestre junio-agosto, el desempleo juvenil pasó de un $18 \%$ al 28\% entre 2019 y 2020.

Los estabilizadores fiscales automáticos, como el seguro de desempleo y el impuesto de renta personal progresivo, sirven para hacer frente al estancamiento o la recesión sin una intervención casuística de las autoridades. Bien diseñados, beneficiarían en mayor grado a los territorios más afectados por el desempleo y la caída de ingresos. Pero en nuestro país, dichos estabilizadores no existen o tienen una importancia secundaria.

\section{Gráfica 2}

Indicador de seguimiento de la economía

Corregido por efecto estacional y de calendario

(Tasa de crecimiento anual)

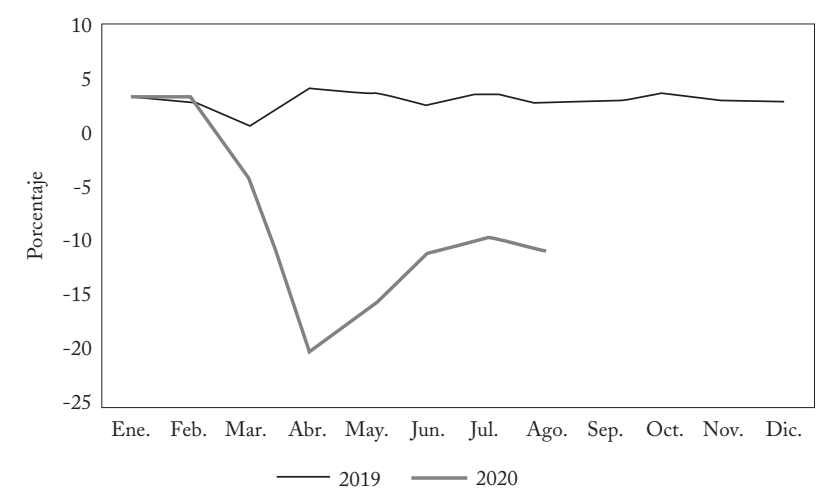

Fuente: Dane [www.dane.gov.co/index.php/estadisticas-por-tema/cuentas-nacionales/indicador-deseguimiento-a-la-economia-ise]. 
E1 producto agregado se desplomó en abril, a juzgar por el Indicador de Seguimiento de la Economía (ISE) del Dane. En ese mes disminuyó un $20 \%$ en términos reales, con respecto a abril de 2019, hasta ahora la caída más pronunciada durante la pandemia (gráfica 2). Desde entonces ha registrado una leve recuperación, y se espera que continúe en lo que resta del año. No obstante, el retroceso anual es inevitable.

\section{ANÁLISIS DEL ESPACIO FISCAL}

Keynes argumentó que en épocas de alto desempleo y contracción económica el Estado debía seguir una política fiscal expansiva. La regla fiscal, adoptada en Colombia mediante la Ley 1473 de 2011, exigía que el gobierno redujera en forma gradual su déficit estructural a $1 \%$ del PIB, o menos, en 2022. Con acierto, el Comité Consultivo del ramo aprobó la suspensión de la regla fiscal durante 2020 y 2021.

Pero ampliar el espacio fiscal no es lo mismo que utilizarlo, o usarlo adecuadamente. En el Marco Fiscal de Mediano Plazo el déficit fiscal previsto para 2020 es del 8,2\% del PIB, un $27 \%$ del cual corresponde al déficit autorizado por el Comité de la Regla Fiscal antes del brote de la enfermedad. E1 40\% es imputable a la caída del recaudo tributario y de otros ingresos asociada a la COVID-19, un efecto automático -no resultado de la intervención de las autoridades- que amortigua el golpe sufrido por el ingreso de los agentes económicos. E1 33\% restante, equivalente al 2,7\% del PIB, sí es atribuible a decisiones tomadas por las autoridades fiscales durante la coyuntura; así, ese 2,7\% del PIB es una medida aproximada del espacio fiscal que, al menos hasta ahora, el gobierno Duque dice querer utilizar para enfrentar la pandemia.

Si en 2020 el déficit fiscal llega al 8,2\% del PIB, el endeudamiento público sería del 65,6\% del PIB (Minhacienda, 2020a). Para que el pago de la deuda no se convierta en un problema en el mediano plazo, el nivel del empleo y del producto debe crecer de manera significativa. Es improbable que esto ocurra bajo la política fiscal actual. El gobierno del presidente Duque ha dado muestras de creer que una carga impositiva baja desata un aumento de la inversión privada. Pero esa concepción subvalora el hecho de que la inversión privada requiere más y mejores bienes y servicios colectivos, cuya provisión está muy rezagada en país.

Siguiendo la política de austeridad, la meta del gobierno es reducir el déficit fiscal al 5,1\% en 2021. E1 proyecto de Presupuesto General de la Nación (PGN) prevé un gasto público equivalente al 21\% del 
PIB en 2021, apenas un leve incremento con respecto al de 2020 (el $20,7 \%$ del PIB). E1 PGN proyecta un aumento no despreciable de la inversión pública en 2021, en infraestructura de transporte, construcción de vivienda y agua potable. Sin embargo, mientras que la inversión aumentaría del 4,3\% al 4,7\% del PIB entre 2020 y 2021, los gastos de funcionamiento se reducirían, de modo que el efecto neto del gasto público sobre el nivel de empleo será probablemente modesto.

\section{FUENTES, USOS Y DEFICIENCIAS DE INFORMACIÓN}

El cuadro 1 muestra los recursos asignados por el gobierno nacional para este año en el marco del estado de emergencia, clasificados por fuentes y usos. E1 monto total asciende a cerca de 29 billones de pesos, un 2,8 \% del PIB. Por fuentes, el $88 \%$ de este monto se destina al Fondo de Mitigación de Emergencias (FOME) y el $11 \%$ al Fondo Nacional de Garantías.

Cuadro 1

Recursos asignados por el gobierno nacional en el marco del Estado de Emergencia por la pandemia de COVID-19

(Billones de pesos corrientes)

\begin{tabular}{lccc}
\hline Fuentes & Monto & $\begin{array}{c}\text { Participa- } \\
\text { ción }(\%)\end{array}$ & $\begin{array}{c}\text { Porcentaje } \\
\text { del PIB }\end{array}$ \\
\hline Fondo Mitigación de Emergencias (FOME) & $\mathbf{2 5 , 5}$ & $\mathbf{8 8 , 3}$ & $\mathbf{2 , 4 9}$ \\
Fondo de Ahorro y Estabilización (FAE) & 12,1 & 41,9 & 1,18 \\
Fondo de Pensiones Territoriales (FONPEP) & 3,0 & 10,4 & 0,29 \\
Títulos de solidaridad & 9,8 & 33,9 & 0,96 \\
Fondo de Riesgos Laborales (FRL) & 0,3 & 1,1 & 0,03 \\
Impuesto solidario funcionarios y contratistas estatales & 0,3 & 1,0 & 0,03 \\
Fondo Nacional de Garantías (FNG) & $\mathbf{3 , 3}$ & $\mathbf{1 1 , 2}$ & $\mathbf{0 , 3 2}$ \\
Fondo Especial Cuota de Fomento de Gas Natural & $\mathbf{0 , 1}$ & $\mathbf{0 , 4}$ & $\mathbf{0 , 0 1}$ \\
\hline Total fuentes & 28,9 & 100,0 & 2,82 \\
\hline Usos & & & \\
\hline Recursos desembolsados o comprometidos & $\mathbf{1 4 , 7}$ & $\mathbf{5 0 , 9}$ & $\mathbf{1 , 4 3}$ \\
FOME & 3,2 & $\mathbf{1 1 , 1}$ & 0,31 \\
Salud & 7,4 & 25,6 & 0,72 \\
Hogares & 4,1 & 14,2 & 0,40 \\
Ingreso solidario & 3,3 & $\mathbf{1 1 , 4}$ & 0,32 \\
Otros (p. ej., Familias acción y Colombia mayor) & 3,3 & 11,4 & 0,32 \\
Empleo & 0,8 & 2,8 & 0,08 \\
Otros & $\mathbf{1 , 3}$ & $\mathbf{4 , 5}$ & $\mathbf{0 , 1 3}$ \\
Fondo Nacional de Garantías (FNG) & $\mathbf{0 , 0}$ & $\mathbf{0 , 0}$ & $\mathbf{0 , 0 0}$ \\
Fondo Especial Cuota De Fomento De Gas Natural & & & \\
Recursos por desembolsar o sin comprometer & $\mathbf{1 0 , 8}$ & $\mathbf{3 7 , 5}$ & $\mathbf{1 , 1}$ \\
FOME & $\mathbf{2 , 0}$ & $\mathbf{6 , 7}$ & $\mathbf{0 , 2}$ \\
Fondo Nacional de Garantías (FNG) & $\mathbf{0 , 1}$ & $\mathbf{0 , 4}$ & $\mathbf{0 , 0}$ \\
Fondo Especial Cuota de Fomento de Gas Natural & $\mathbf{2 8 , 9}$ & $\mathbf{1 0 0 , 0}$ & 2,82 \\
\hline Total usos & & &
\end{tabular}

Nota: los datos más recientes son del 9 de octubre de 2020. El PIB se estimó suponiendo un crecimiento del $-5,5 \%$ y una inflación del $2 \%$ en 2020.

Fuente: Minhacienda [minhacienda.gov.co/webcenter/portal/MedidasCovid-19/pages_medidas-Covid19] y Observatorio Fiscal de la Universidad Javeriana [www.ofiscal.org/gastos-fome]. 
El cuadro 1 también muestra el uso de los recursos por grandes rubros: de los 29 billones asignados, el 56,7\% se ha comprometido o desembolsado, con destino a hogares, empleo y salud, en ese orden. En salud no fue posible determinar cuánto se ha dedicado a la ampliación de la oferta hospitalaria o de la red pública existente. El programa que ha ejecutado más recursos es Ingreso Solidario.

El ministro de Hacienda ha aclarado que la ejecución de algunos programas implica el giro efectivo de recursos, como los programas de Apoyo al Empleo Formal e Ingreso Solidario; y que la de otros consiste en otorgar garantías de crédito, que pueden o no ocasionar desembolsos, como los programas del Fondo Nacional de Garantías.

\section{PROGRAMAS: ALCANCES Y CUESTIONAMIENTOS}

Las características de los programas que el gobierno ha puesto en marcha durante el estado de emergencia y su ejecución llaman a ser cautelosos con respecto a su eficacia. E1 Programa de Apoyo al Empleo Formal (PAEF), que busca otorgar un subsidio mensual a empresarios formales para pagar la nómina -un $40 \%$ del salario mínimo por trabajador-, debería beneficiar en últimas a los obreros y empleados particulares. Según la Unidad de Pensiones y Parafiscales, hasta el 25 de octubre el total girado por este subsidio era de 3,1 billones de pesos. Como porcentaje del PIB, este valor es pequeño (un 0,3\%), con respecto al peso relativo de obreros y empleados particulares en la fuerza laboral.

El programa Ingreso Solidario, creado en abril como un mecanismo pasajero para entregar "una transferencia monetaria en favor de los hogares en situación de pobreza y vulnerabilidad que no sean beneficiarios de programas sociales del Estado" (DNP, 2020), ha tenido grandes problemas para identificar a los beneficiarios y girar los recursos. Con una duración inicial de tres meses, prorrogable (de hecho, se prorrogó hasta marzo de 2021), el programa prevé transferir 160.000 pesos mensuales por beneficiario.

Según el DNP, el número de hogares beneficiados por Ingreso Solidario supera los 2,6 millones. La suma total transferida hasta finales de octubre sería de unos 4 billones, un 0,4\% del PIB. Este programa se dirige a trabajadores por cuenta propia que, por el aislamiento obligatorio u otras razones, no han podido trabajar. Y podría ser la semilla de programas de renta básica o seguro de desempleo, que morigerarían automáticamente las fluctuaciones del nivel de ingresos y de empleo en departamentos y regiones. 
En tal sentido se presentó el proyecto de Ley 310 de 2020 en el Senado de la República encaminado a crear un "Programa de renta básica de emergencia", que sustituya a Ingreso Solidario. Esa renta básica sería una transferencia no condicionada del gobierno nacional, equivalente a un salario mínimo mensual legal, a "los hogares en situación de pobreza y vulnerabilidad, así como de los trabajadores en micronegocios por un periodo de tres (3) meses". Sería un programa focalizado, al que se ha cuestionado su carácter transitorio y, de prolongarse, su costo. Investigadores de la Universidad de los Andes proponen transferir a las personas de bajos ingresos una suma que complete el ingreso básico fijado por la legislación (GIM, 2020). En comparación con el proyecto de Ley del Senado, este programa, llamado Ingreso Básico Garantizado, tendría una focalización más afinada (personas en vez de hogares), sería menos costoso periodo a periodo (se transferiría el faltante para completar el ingreso básico, en una de sus versiones equivalente a la línea de pobreza y no al salario mínimo) y sería permanente. Con el fin de determinar la suma de la transferencia se establecería la declaración de renta obligatoria para todos los adultos. He ahí una gran debilidad de esta propuesta, pues la probabilidad de que no se presente declaración es mayor entre personas más pobres (p. ej., analfabetas, vendedores ambulantes, sin techo), la población objetivo del ingreso básico.

Ante la amenaza de la crisis a la supervivencia de las empresas, el gobierno of reció garantizar el endeudamiento de las pequeñas y medianas empresas para pagar de nómina y financiar capital de trabajo. E1 respaldo se extendió a las microempresas y los trabajadores independientes. El monto asignado a estos programas de garantías (17,2 billones de pesos) corresponde a cupos anunciados. A finales de octubre las entidades financieras solo habían desembolsado el 51\% del crédito respaldado por esas garantías.

La eficacia del endeudamiento para financiar gastos corrientes de las empresas y darles liquidez es dudosa. El porcentaje de garantías es relativamente alto (entre el 70 y el 90), pero los empresarios y banqueros pueden ser reticentes a recurrir a ese expediente.

Otra iniciativa que ha sido cuestionada es que el gobierno nacional destine recursos para salvar empresas privadas. En principio, son preferibles las políticas públicas de carácter general, horizontales o sectoriales, e imparciales entre empresas. Pero se pueden canalizar recursos a la empresa privada para minimizar las pérdidas de empleo, como se ha venido haciendo con los subsidios para pagar nómina. Con todo, no es justificable seleccionar en forma arbitraria a ganadores o 
perdedores específicos. El eventual salvamento solo procedería si el Estado recibe garantías y contraprestaciones suficientes, bien sea en forma de propiedad accionaria o de protección o creación de empleos.

Aunque la eficacia de las políticas económicas es limitada ante la magnitud de la crisis, para los empresarios y trabajadores que perdieron su fuente de ingresos por la pandemia, los programas de apoyo llegaron demasiado tarde o fueron inadecuados o insuficientes.

Más allá del corto plazo, se debe dotar al Estado de una mejor caja de herramientas para encarar crisis socioeconómicas y desastres naturales, en áreas como el sistema de salud, la seguridad alimentaria, la protección del empleo y los estabilizadores fiscales automáticos. $\mathrm{E}$ incluir en ellas algún tipo de ingreso básico permanente.

\section{¿UNA NUEVA REFORMA TRIBUTARIA?}

El recaudo tributario en el país ha sido históricamente bajo: hasta mediados del siglo XX, los impuestos del orden nacional no superaron el 4\% del PIB, y en 1983 apenas rondaban el 6\% (Avella, 2009). Con la consolidación del IVA, a mediados de la década de 1980, empezó a crecer la tributación nacional: el recaudo pasó a representar el 14\% del PIB en el año 2000, un porcentaje que se mantiene desde entonces. Hoy la carga tributaria es algo inferior a la de países similares en ingreso per cápita y muy inferior a la de los países desarrollados.

Desde que se expidió la Constitución de 1991, los gastos permanentes del gobierno nacional han sido mayores que los ingresos permanentes; de ahí que en la agenda política suele contemplar una reforma que eleve el nivel de tributación. Una reforma de ese tipo, que ya era difícil de realizar antes de la pandemia, afronta una nueva dificultad: los efectos económicos adversos de adoptarla en medio de una recesión profunda. Habría una salida: se podría orientar a gravar más la riqueza que el ingreso, y más el ingreso que el consumo. Ello implicaría dar prelación a gravámenes como el impuesto al patrimonio y el de renta personal. Gravar en forma progresiva las grandes riquezas y los altos ingresos no frenaría, o no tanto, la recuperación económica, y favorecería la equidad.

Pero en vez de acudir a tributos probados como los impuestos al patrimonio y a los dividendos, que se pueden aumentar y calibrar para gravar a los ricos en forma progresiva y no confiscatoria, en los afanes creados por la pandemia se han recetado paliativos improvisados y caritativos para obtener recursos adicionales. Por ejemplo, que los congresistas donen un mes de salario o que los empleados públicos 
y contratistas del Estado que devengan más de 10 millones de pesos aporten, por tres meses, el 10\% de sus remuneraciones. Esto paliativos encuentran su parangón en la esfera privada: "La asamblea de accionistas del Grupo X, reunida en forma virtual, aprobó distribuir dividendos por 1,34 billones de pesos"; según la prensa, el principal accionista del conglomerado anuncia que "donará 80.000 millones para enfrentar el coronavirus". Se trata de una donación por una sola vez que equivale al $6 \%$ de los dividendos distribuidos (como referencia, en 2019 el sector financiero tuvo unas utilidades de 17 billones de pesos). Pese a que son contribuciones importantes, la caridad no puede sustituir a los impuestos permanentes. Pasados los afanes iniciales, hay indicios de que, para los actuales diseñadores de política, reforma tributaria estructural significa tratamiento tributario más favorable a los ingresos de capital.

Las crisis socioeconómicas y los desastres naturales suelen ser recurrentes, aunque no periódicas. Ante la COVID-19, el gobierno nacional solo ha adoptado momento medidas ad hoc, transitorias, $\mathrm{y}$ encaminadas únicamente a enfrentar sus efectos inmediatos. Pese a la complejidad de la situación, pese a la incertidumbre sobre el futuro, queda la pregunta inquietante de cuánto se hablará la próxima vez de falta de providencia.

\section{REFERENCIAS BIBLIOGRÁFICAS}

Avella, M. (2009). El crecimiento del gasto público en Colombia: 19252003, ¿una visión descriptiva a la Wagner o a la Peacok y Wiseman? Revista de Economía Institucional, 11(20), 83-137.

Cede. (2020). Efectos en pobreza y desigualdad del Covid-19 en Colombia: un retroceso de dos décadas. Nota Macroeconómica, 20, Bogotá: Universidad de los Andes.

CID. (2020). Covid-19: efectos económicos y políticas públicas para enfrentar la crisis. Investigaciones, 36, [www.fce.unal.edu.co/].

Dane. (2020a). Estadísticas Vitales - EEVV. Boletin excepcional COVID-19, 16 de octubre, [www.dane.gov.co].

Dane. (2020b). Pobreza monetaria en Colombia. Actualización metodológica, 13 de octubre, [www.dane.gov.co].

DNP. (2019). Bases del Plan Nacional de Desarrollo 2018-2022. Bogotá, D.C.: DNP.

DNP. (2020). Ingreso Solidario, [https://ingresosolidario.dnp.gov.co/].

Farné, S., Rodríguez, D. y Ríos, P. (2017). Protección económica para la vejez en Colombia. Páginas de Seguridad Social, 1(1), 93-121.

Gómez, J. E. (2020). Hacia una política pública de garantía del trabajo. Dinero, [https://www.dinero.com/opinion/columnistas/articulo/ hacia-una-politica-publica-de-garantia-de-trabajo-por-jose-eduardogomez/282226]. 
GIM. (2020). Ingreso básico garantizado (IBG): ¿es el momento ideal de implementarlo? Nota Macroeconómica, 22, Bogotá; Universidad de losAndes.

Keynes, J. M. (1965). Teoría general de la ocupación, el interés y el dinero [1936]. México DF: Fondo de Cultura Económica.

Kolata, G. (2020). How pandemics end. The New York Times, [www. nytimes.com].

Minhacienda. (2020a). Marco fiscal de mediano plazo 2020. Bogotá, D.C.: Ministerio de Hacienda.

Minhacienda. (2020b). Mensaje presidencial Proyecto de ley Presupuesto General de la Nación 2021. Bogotá, D.C.: Ministerio de Hacienda.

Ocampo, J. A. (2020). From the Washington to the Latin American Consensus. [www.brookings.edu].

Okun, A. (1962). Potential GNP: Its measurement and significance. Cowles Foundation Paper (190).

Peacock, A. T. y Wiseman, J. (1961). The growth of public expenditure in the United Kingdom. Princeton: Princeton University Press.

Robinson, J. (1978). Obstacles to full employment. En Contributions to modern economics (pp. 20-28). Nueva York: Academic Press.

Sayed, A., y Peng, B. (2020). Pandemics and income inequality: A historical review. Covid Economics, 52, 96-117.

Wray, L. R. (2007). The employer of last resort programme: Could it work for developing countries? Economic and Labour Market Papers, Ginebra: ILO. 